\title{
CARACTERÍSTICAS DOS GRUPOS DE PESQUISA DA ENFERMAGEM BRASILEIRA CERTIFICADOS PELO CNPQ DE 2005 A 2007
}

\author{
Research Group Characteristics of the Brazilian Nursing \\ Certificated by the CNPq from 2005 to 2007 \\ Caracteristicas de los Grupos de Investigación de la Enfermería \\ Brasileña Certificados por el CNPq de 2005 hasta 2007
}

\begin{abstract}
Resumo
Trata-se de um estudo documental, constituindo-se um banco de dados coletados em quatro momentos distintos: março de 2005, dezembro de 2005, agosto de 2006 e abril de 2007, dados estes referentes ao total de Grupos de Pesquisa de Enfermagem encontrados no Diretório dos Grupos de Pesquisa no Brasil do CNPq. Os resultados indicam que $92 \%$ dos grupos de pesquisa possuem de 1 a 5 linhas de pesquisa, são compostos por 11 a 15 membros, oscilando em uma média de até 8 pesquisadores, 5 doutores, 3 mestres, 3 especialistas e 7 graduandos. Logo, os grupos de pesquisa representam o lócus de produção de conhecimentos e formação de recursos humanos em pesquisa. No Brasil, houve um crescimento significativo dos grupos de pesquisa da área da Enfermagem com o incremento da produção, qualificação dos integrantes, bem como 0 fortalecimento das bases de investigação e a maior visibilidade e reconhecimento da inovação da Enfermagem.
\end{abstract}

Palavras-chave: Grupos de Pesquisa. Enfermagem. Saúde. Pesquisa em Enfermagem.

\section{Abstract}

This is a documentary study and consisting a data base collected at four distinct moments: march of 2005, December of 2005, august of 2006 and April of 2007, these information are referring to the total of Research Groups of Nursing in the Directory of Research Groups of in Brazil of the CNPq. The results indicate that $92 \%$ of the research groups possess of 01 - 05 research lines, are composites of 11 - 15 members, oscillating in a average of 08 researchers, 05 doctors, 03 masters, 03 specialists, 07 student of graduation. Soon, the research groups are center of production of knowledge and formation of human resources in research. In Brazil, it had a significant growth of the research groups Nursing with the increment of the production, qualification of the integrants, the for tification of the inquiry bases and the biggest visibility and recognition of innovation of the Nursing.

Keywords:

Research Groups. Nursing. Health. Nursing Research.

\section{Resumen}

Estudio documental que constituye en una base de datos recogida en cuatro momentos distintos: marzo de 2005, diciembre del 2005 , agosto de 2006, y abril de 2007, datos éstos que refieren el total de grupos de investigación encontrados en el directorio de los grupos de investigación en el Brasil del CNPq. Los resultados indican que los $92 \%$ de los grupos de investigación poseen de 01 las 05 líneas de la investigación, son compuestos para 11 los 15 miembros, oscilando en un promedio de hasta 08 investigadores, 05 doctores, 03 masteres, 03 especialistas, 07 graduandos. Luego, los grupos de investigación se constituyen en el locus de la producción del conocimiento y de la formación de recursos humanos en la investigación. En el Brasil, hubo un crecimiento significativo de los grupos de investigación del área de la enfermería con el incremento de la producción, la calificación de los integrantes, así como, el fortalecimiento de las bases de la investigación y de la mayor visibilidad y reconocimiento de la innovación de la enfermería.

Palabras clave: Grupos de Investigación. Enfermería. Salud. Investigación en Enfermería. 


\section{INTRODUÇÃO}

As atividades de produção de conhecimentos na modalidade de pesquisa vêm sendo desenvolvidas por equipes de pesquisadores titulados ou em formação, organizados sob a designação de Grupos de Pesquisa.

0 Conselho Nacional de Desenvolvimento Científico e Tecnológico (CNPq), criado em 1951, é uma Fundação vinculada ao Ministério da Ciência e Tecnologia (MCT). Destina-se ao apoio à Ciência, Tecnologia e Inovação na formação e absorção de recursos humanos e financiamento de projetos de pesquisa que contribuem para o aumento da produção de conhecimento e geração de novas oportunidades de crescimento para o País 1 . Este órgão cadastra os grupos de pesquisa do país, incluindo os da área da Enfermagem, há mais de 15 anos. Para tanto, mantém um Diretório de Grupos de Pesquisa com o registro das informações disponibilizadas para a sociedade sobre a estrutura dos mesmos, tanto dos emergentes como dos consolidados, atribuindo um selo de certificação.

$\mathrm{Na}$ Enfermagem brasileira estes grupos surgiram na década de 1970, e seus crescimento e forma de organização vêm se expandindo de modo significativo. Atualmente chegam a um total aproximado de 270 grupos cadastrados no Diretório de Pesquisa do CNPq.

Entende-se que o desenvolvimento da pesquisa é uma importante estratégia para o fortalecimento da Enfermagem como ciência e profissão, pois é importante e necessário exercer uma prática profissional sustentada por uma contínua busca de novos conhecimentos. E a competência para cuidar, pesquisando, educando e gerenciando, vem sendo desenvolvida na formação e no exercício profissional do enfermeiro.

A Enfermagem necessita incrementar a produção de conhecimentos através da pesquisa para maior visibilidade, reconhecimento e consolidação da profissão como ciência, tecnologia e inovação. Isto se reflete na sua melhor qualificação do ensino nos níveis de graduação e pós-graduação, o qual orienta-se por uma prática de cuidado responsável com a vida e saúde do cidadão, promovendo o seu viver em melhores condições de saúde.

A competência investigativa é necessária para o desenvolvimento sócio-político e tecnológico em saúde e primordial na formação, na prática e nos centros de investigação da área.

É reconhecida a importância da capacidade de produzir ciência, tecnologia e inovação para a melhoria da saúde da população e avanço no desenvolvimento do país, bem como de contar com instituições adequadas de ensino e formação em ciência e tecnologia.

0 processo de construção de conhecimentos passa pela possibilidade de dispor de recursos humanos competentes no processo investigativo, no domínio dos diferentes métodos de investigação e das bases teórico-filosóficas que sustentam o saber da Enfermagem, bem como na gerência do processo produtivo rumo à excelência ou à qualificação.

No Brasil, a Enfermagem é uma profissão de formação universitária e em nível avançado. Conta atualmente com 32 programas de pós-graduação em Enfermagem credenciados pela CAPES, dos quais 14 possuem o nível de doutorado ${ }^{2}$. A atividade de pesquisa realizada nestes programas de pós-graduação tem contribuído para a criação e consolidação de novas linhas de pesquisa, no crescimento quantiqualitativo das publicações, bem como na melhor qualificação dos graduandos, mestres e doutores da área e no fortalecimento dos grupos de pesquisa. Trata-se de um processo cada vez mais integrado entre alunos e docentes das instituições de ensino com os profissionais das instituições da saúde através dos centros ou grupos de pesquisa.

Os grupos de pesquisa fornecem o suporte necessário de infraestrutura para as atividades de pesquisa dos enfermeiros, graduandos e pós-graduandos, a oportunidade de trabalho integrado e a possibilidade de incremento do potencial em pesquisa.

0 impacto e o benefício econômico e social dos conhecimentos produzidos e aplicados na prática da enfermagem é ainda um grande desafio para os nossos pesquisadores gerentes das práticas investigativas e coresponsáveis nos trabalhos da área da saúde.

A Enfermagem no CNPq passa a ser visível como área de conhecimento a partir da década de 1970 e a ter representante de área desde a década de 1980, chegando hoje a ter seu Comitê Assessor CA-EF (jun. 2006). 0 CA-EF é constituído por três pesquisadoras representantes titulares e uma suplente. Estas reconhecem e testemunham a importância e a evolução da Enfermagem como ciência e tecnologia no CNPq, a importância do fomento para viabilização e incremento das pesquisas na área, os avanços obtidos, os desafios enfrentados e as perspectivas promissoras ao fortalecimento e consolidação da Enfermagem³.

No Brasil, os conhecimentos são organizados em áreas de concentração e linhas de pesquisa. Entende-se por área de concentração um campo delimitado de certo(s) ramo(s) de conhecimento(s), atividade(s) ou competência(s). Refere-se ao universo de disciplinas e interdisciplinas, à organização das mesmas em especialidades ou processo de viver, processo saúde e doença, dentre outras, e à possibilidade de classificação para visibilidade mediante nomenclatura internacional.

Uma área de concentração se sustenta por um domínio temático conformado por linhas de pesquisa. A linha de pesquisa determina os rumos do que vem sendo investigado num dado contexto ou realidade, limita as fronteiras de um campo espećíico de conhecimento, oferece orientação teórica para a busca e estabelece procedimentos adequados ao processo de construção do conhecimento. Uma linha de pesquisa dá idéia de produção circunscrita a um mesmo âmbito temático ou teórico ${ }^{4}$.

Toda produção intelectual do pesquisador/docente situa-se em uma ou alguma linha de pesquisa. As linhas de pesquisa se constituem em unidades de análise de desempenho especialmente dos cursos de graduação e pós-graduação, da produção dos pesquisadores nos órgãos de fomento à pesquisa, e outros.

A indicação do vínculo das produç̃es em linhas de pesquisa ainda mostra pouca clareza da sua determinação, levando a perceber superposição de linhas, imbricação ou interfaces, concentração ou convergência, pulverização ou diversidade de temas, dentre outros. Ainda são noções "frouxas", de múltiplos entendimentos e formas de utilização e registro. Porém, são das linhas de pesquisa que surgem ou derivam os projetos de pesquisas nas suas diferentes modalidades. 
Os projetos de pesquisa são estruturas propositivas de produção de conhecimentos através do processo investigativo e se viabilizam nos grupos de pesquisa. A tendência atual é de produção integrada e em parcerias, e não mais de projetos isolados e realizados apenas um de cada vez. Assim, o grupo de pesquisa acaba sendo a forma de organização adequada para a realização de atividades coletivas ou compartilhadas de produção de conhecimentos. Todavia, como se trata de uma modalidade um tanto recente, corre o risco de ser pulverizado ou, ao contrário, super dimensionado, prejudicando a sua produtividade. Uma vez bem organizado, com normas de controle, o mesmo pode servir de instrumento de fomento. Porém ainda faz-se necessário avançar na compreensão do que é um grupo de pesquisa para a área, quais são as suas principais características, bem como os critérios específicos da área para análise do mérito no seu credenciamento, hoje nas modalidades de emergentes e consolidados.

No site do CNPq, www.cnpq.br/diretório dos grupos de pesquisa ${ }^{5}$, pode-se encontrar resposta para as seguintes questões: 0 que é o Diretório dos Grupos de Pesquisa? Para que serve? Como é feita a aquisição dos dados do Diretório? Como é disponibilizado o acesso às bases de dados e aos sistemas de coleta e atualização de dados do Diretório? Quais os principais objetivos do Diretório? Quais informações estão contidas nas bases de dados do Diretório? Como são obtidas as informações constantes do Diretório? 0 que é um grupo de pesquisa? Como saber se as atividades desenvolvidas por um conjunto de pesquisadores constituem um grupo de pesquisa? 0 que é linha de pesquisa? Qual a diferença entre linha e projeto de pesquisa? Quem é um líder de grupo? Quem são os pesquisadores de um grupo? Quem são os estudantes de um grupo? Como são identificados os líderes de grupos de pesquisa? Como são identificados os pesquisadores, estudantes e técnicos de um grupo? Quais são as instituições participantes do Diretório dos Grupos de Pesquisa? Quais os procedimentos para inclusão de uma nova instituição no Diretório dos Grupos de Pesquisa? Como ter acesso aos dados das versões anteriores do Diretório dos Grupos de Pesquisa? Onde encontrar informações/ orientações sobre a versão atual (5.0) do Diretório? Dentre as respostas das questões acima ${ }^{5}$, destacamos algumas:

0 grupo de pesquisa é definido como um conjunto de indivíduos organizados hierarquicamente em torno de uma ou, eventualmente, duas lideranças: o fundamento organizador dessa hierarquia é a experiência, o destaque e a liderança no terreno científico ou tecnológico; são envolvidos profissional e permanentemente com atividades de pesquisa, cujo trabalho se organiza em torno de linhas comuns de pesquisa; em algum grau, compartilham instalações e equipamentos. 0 conceito de grupo admite aquele composto de apenas um pesquisador. Na quase totalidade desses casos (cerca de 10\% do total de grupos, na experiência de 10 anos do Diretório), os grupos se compõem do pesquisador e de seus estudantes ${ }^{(5)}$.

Linhas de pesquisa representam temas aglutinadores de estudos científicos que se fundamentam em tradição investigativa, de onde se originam projetos cujos resultados guardam afinidades entre si. Projeto de pesquisa é a investigação com início e final definidos, fundamentada em objetivos específicos, visando à obtenção de resultados, de causa e efeito, ou à colocação de fatos novos em evidência 5 .

0 pesquisador líder de grupo é o personagem que detém a liderança acadêmica e intelectual naquele ambiente de pesquisa. Normalmente, tem a responsabilidade de coordenação e planejamento dos trabalhos de pesquisa do grupo. Sua função aglutina os esforços dos demais pesquisadores e aponta horizontes e novas áreas de atuação dos trabalhos ${ }^{5}$.

Pesquisadores são os membros graduados ou pósgraduados da equipe de pesquisa direta e criativamente envolvidos com a realização de projetos e com a produção científica, tecnológica e artística do grupo. Estagiários pós-doutores são considerados pesquisadores 5 .

São estudantes (bolsistas ou não) em iniciação científica e pós-graduação (exceto os estagiários em nível de pós-doutoramento, que devem ser considerados pesquisadores), que participam ativamente das linhas de pesquisa desenvolvidas pelo grupo, como parte de suas atividades discentes, sob a orientação de pesquisadores do grupo ${ }^{5}$.

Diante das considerações apresentadas, levantam-se alguns questionamentos em relação aos grupos de pesquisa na Enfermagem brasileira. Dentre eles, as modalidades de organização do trabalho dos pesquisadores (isolado, compartilhado ou ambos), a importância dos mesmos para a produção de conhecimentos (PG: M, D, PD; E; G; instituiç̃̃es da saúde, outras), o que produziram, a relevância desta produção, áreas e linhas de pesquisa vinculadas, denominação e visibilidade da Enfermagem nos sistemas de informação (cadastro, certificado), localização (instituiç̧̃es envolvidas), organização (pessoas, competências/titulação, atribuições, instrumentos normativos), infra-estrutura física e material utilizada, tipos de atividades que desenvolvem (pesquisa, ensino, orientação, extensão e outras; dinâmica de funcionamento), abrangência de atuação (local, regional, nacional e internacional; intercâmbios, parcerias, atividades compartilhadas), fatores que auxiliam ou dificultam a produtividade dos mesmos, políticas de apoio/incremento da produção, perspectivas de avanços, estudos já realizados sobre os mesmos, e outros.

Ainda carecemos de estudos que mostrem o conhecimento sobre a composição dos grupos da área da Enfermagem, sua vinculação institucional, operacionalização das pesquisas, suas potencialidades, limitações e fragilidades, dentre outros aspectos, com vista a traçar um plano nacional de fortalecimento dessas células de pesquisa.

$\mathrm{Na}$ condição de representantes da área da Enfermagem no CNPq a partir de 2004 e com o apoio do trabalho da bolsista de iniciação científica, sob minha orientação, de igual período de vigência da bolsa, 2004-7, realizamos um banco de dados dos grupos de pesquisa da área para compor informações que 
pudessem ser apresentadas em reuniões e eventos da área. Este banco de dados possibilita dar resposta a algumas das questões aqui levantadas, com o objetivo de descrever as principais características dos grupos de pesquisa da área da Enfermagem cadastrados no Diretório de Grupos de Pesquisa do CNPq.

\section{METODOLOGIA}

Realizou-se um estudo documental, constituindo-se um banco de dados coletados em quatro momentos distintos: março de 2005, dezembro de 2005, agosto de 2006 e abril de 2007, dados estes referentes ao total de grupos de pesquisa de Enfermagem encontrados no Diretório dos Grupos de Pesquisa no Brasil, que são bases de dados (bases censitárias: censo 2004; e base corrente) que contêm informações sobre os grupos de pesquisa em atividade no País ${ }^{6}$. Estes estão disponíveis no site do CNPq, no linkBuscas, na busca textual por Enfermagem. Os grupos de pesquisa objetivados para análise de dados constituíam base de dados pertencente à área predominante da Enfermagem, sendo excluídos os diversos grupos de outras áreas.

A primeira coleta de dados ocorreu em março de 2005, na qual se localizaram 234 grupos de pesquisa na área da Enfermagem e 40 grupos de pesquisa que pertenciam a outras áreas predominantes, muito embora relacionassem seus estudos com a área da Saúde e a Enfermagem. Dentre todos os encontrados, foram selecionados apenas os que apresentavam Enfermagem como área predominante. Nessa primeira coleta, a busca exigia apenas o preenchimento de um campo com a palavra Enfermagem. Na segunda coleta, realizada em dezembro de 2005, o site do CNPq fora encontrado com modificações; além de inserir a palavra Enfermagem no campo de busca textual, era preciso assinalar alguns campos que tornam a busca mais abrangente ou específica de acordo com a intenção do pesquisador. Foram selecionados os itens: nome do GP, nome da linha de pesquisa do GP, repercussões do GP, palavra-chave de linha de pesquisa e nome do pesquisador, acreditando-se que nesses tópicos dos grupos de pesquisa em Enfermagem fosse citada a palavra Enfermagem. Nesta coleta foram localizados 340 grupos de pesquisa. Destes, somaram-se 251 grupos de pesquisa de Enfermagem, e 89 grupos pertenciam a áreas predominantes diferentes. Na terceira coleta, desenvolvida no mês de agosto de 2006, o site manteve-se com a mesma configuração anterior e a busca resultou nos mesmos números da segunda coleta, ou seja, 340 grupos de pesquisa, dos quais 251 de Enfermagem e 89 pertencentes a outras áreas predominantes; porém, nestes foram observadas alterações nas informações fornecidas em cada grupo em relação a coleta anterior. Uma última coleta foi feita em abril de 2007, com busca textual na base corrente para uma nova verificação sobre a situação dos grupos de pesquisa de Enfermagem nesta data, resultando na identificação agora de 270 grupos de pesquisa de Enfermagem como área predominante.

Os elementos destacados para análise sobre cada grupo de pesquisa foram: instituições, ano de formação dos grupos de pesquisa, linhas de pesquisa, número de integrantes, de estudantes, de técnicos, de pesquisadores. 0 número de doutores, mestres, especialistas e graduandos foram referentes apenas à coleta do mês de dezembro de 2005 e agosto de 2006, pois só neste período que foram disponibilizadas estas informações.

Os dados obtidos foram agrupados em forma de tabelas para melhor visualização de sua representação.

\section{RESULTADOS}

Na buscas dos grupos de pesquisa da área da Enfermagem depara-se com a prática interdisciplinar dos conceitos e conhecimentos no campo da saúde e enfermagem. A necessidade de adotar diferentes abordagens para compreender a realidade e enfrentar os problemas que se apresentam tornam a saúde um processo dinâmico e complexo, "cuja compreensão aponta a reflexões interdisciplinares para uma nova prática em saúde e enfermagem"7.

Esta nova forma de abordagem se reflete na Enfermagem na denominação dos seus vários grupos de pesquisa de outras áreas do conhecimento cadastrados no Diretório dos Grupos de Pesquisa do CNPq. Entre eles se apresentam os conhecimentos das áreas ou campos como: Administração, Antropologia, Bioquímica, Ciências da Computação, Ciências Políticas, Economia, Educação, Educação Física, Farmácia, Filosofia, Genética, História, Letras, Medicina, Nutrição, Odontologia, Psicologia, Saúde Coletiva, Serviço Social, Sociologia, dentre outros. Assim, o uso de descritores ou as denominações mais específicas da área da Enfermagem podem não ser suficientes para rastrear todos os grupos de pesquisas que realmente pertencem ou se efetivam dentro da área da Enfermagem. Um expressivo número de grupos de pesquisa da Enfermagem com importante produção na própria área não indicam na sua denominação a palavra "enfermagem", o que leva a se perder significativo volume de produções e, ao mesmo tempo, não dar a visibilidade real do que a área de Enfermagem tem, produz ou realiza no campo da produção de conhecimentos.

0 fato de a Enfermagem ter sido citada em outras áreas predominantes, com destaque para a Saúde Coletiva e Medicina, denota um avanço na ampliação do conhecimento, procurando uma integração do pensamento por meio de troca de informação, justaposição de conteúdos, visando à qualidade do desenvolvimento das mais diversas tecnologias em prol da saúde humana. Pois "uma das finalidades da Universidade é a produção do conhecimento que dará impulso ao desenvolvimento social, econômico e tecnológico de uma comunidade. Para que isso ocorra é preciso entender como o conhecimento se articula nesta dinâmica"

Pelos dados do Diretório dos Grupos de Pesquisa do CNPq, temos que o surgimento do primeiro grupo de pesquisa em Enfermagem foi no ano de 1973, o Núcleo de Estudos e Pesquisas do Idoso - NESP/UFBA. E apenas nove anos depois, em 1982, é criado o Grupo de Estudos sobre Cuidado de Saúde de Pessoas Idosas - GESPI/UFSC, seguido pelo Núcleo de Estudos da Criança e do Adolescente/UNIFESP.

Desde 1973 até abril de 2007 percebe-se o aumento para 270 grupos de pesquisa em Enfermagem, com os valores oscilando de acordo com o período da coleta de dados, conforme demonstrado na tabela 1.

Os grupos de pesquisa crescem à medida que a pósgraduação em Enfermagem no Brasil se amplia. Porém, em 
Tabela 1: Ano de formação dos Grupos de Pesquisa em Enfermagem no período de 2005 - 2007.

\begin{tabular}{|c|c|c|c|c|}
\hline $\begin{array}{l}\text { Ano de formação } \\
\text { dos GP }\end{array}$ & 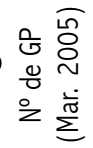 & 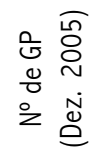 & 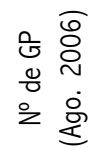 & 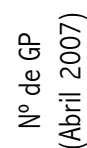 \\
\hline $1973-1977$ & 1 & 1 & 1 & - \\
\hline $1978-1982$ & 2 & 2 & 2 & 2 \\
\hline $1983-1987$ & 8 & 10 & 7 & 9 \\
\hline $1988-1992$ & 28 & 27 & 28 & 26 \\
\hline $1993-1997$ & 56 & 58 & 62 & 54 \\
\hline $1998-2002$ & 90 & 108 & 104 & 82 \\
\hline $2003-2007$ & 49 & 45 & 47 & 97 \\
\hline
\end{tabular}

Fonte: Base de Dados do Diretório dos Grupos de Pesquisa CNPq.

relação à divulgação, os caminhos mais usados para comunicação no meio científico pelos enfermeiros são os congressos, simpósios e reuniões científicas, sendo reduzido o número de publicações em periódicos científicos. Dessa forma, a pesquisa em Enfermagem no Brasil expande-se de forma progressiva, e a lenta difusão do conhecimento pode ser decorrente dos canais utilizados pelos enfermeiros ${ }^{9}$.

A sociedade contemporânea tem sido desafiada a caminhar para a construção de uma universidade compatível com a exigência dos novos tempos, e os cursos de pós-graduação têm proporcionado a aproximação de referenciais teóricos e práticos que venham a subsidiar a prática educativa e a dar suporte para a necessária busca de transformações e a construção do conhecimento ${ }^{10}$.

Partindo da perspectiva de que a maioria dos países do chamado eixo sul, incluindo o Brasil, são colocados na posição de consumidores das tecnologias produzidas nos países do eixo norte do planeta, evidencia-se a necessidade da concretização de uma política científica que promova o domínio científico e tecnológico para a soberania do país e maior competitividade das empresas nacionais ${ }^{11}$.

Os investimentos feitos são direcionados para a formação e absorção de recursos humanos e financiamento de projetos de pesquisa que contribuam para 0 aumento da produção de conhecimento e geração de novas oportunidades de crescimento para o país ${ }^{1}$.

Essa produção de conhecimentos é constituída por artigos em periódicos científicos indexados, livros, capítulos de livros, resumos, outras publicações, patentes, aperfeiçoamento científico dos profissionais, teses de doutoramento e dissertações de mestrado. 0 reflexo da busca de novos talentos na iniciação científica e do apoio ao trabalho de pesquisador produtividade em pesquisa, o apoio aos grupos de pesquisa, 0 fortalecimento das linhas de pesquisa e a viabilização de políticas e prioridades em pesquisa foram criados na soma do conhecimento em Enfermagem. No entanto, esta produção ainda depende da demanda de projetos versus fomento, políticas e estratégias das instituições de ensino superior, programas de pós-graduação e cursos de graduação, sociedades científicas, grupos de pesquisa, entre outros.
Com relação às linhas de pesquisa com que os grupos de pesquisa são configurados, hodiernamente $92 \%$ dos grupos de pesquisa possuem 1 a 5 linhas de pesquisa, o que demonstra uma área de concentração bastante direcionada entre seus membros, conforme tabela abaixo:

Tabela 2: Quantidade de Linhas de Pesquisa dos Grupos de Pesquisa em Enfermagem do Brasil no período de 2005 - 2007.

\begin{tabular}{|c|c|c|c|c|}
\hline $\begin{array}{l}N^{0} \text { de linhas } \\
\text { de pesquisa }\end{array}$ & 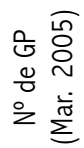 & 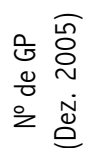 & 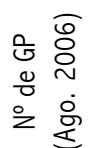 & 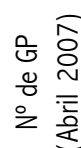 \\
\hline $1-5$ & 214 & 231 & 232 & 250 \\
\hline $6-10$ & 17 & 17 & 16 & 18 \\
\hline $11-15$ & 3 & 3 & 3 & 2 \\
\hline $16-20$ & - & - & - & - \\
\hline
\end{tabular}

A atual tendência da pós-graduação no Brasil caracterizase pelo incentivo e direcionamento da produção do conhecimento às linhas de pesquisa dos grupos de pesquisa, sistematizando a produção científica. Linhas de pesquisa referem-se

Fundamentalmente a campos problematizadores que exigem estudos aprofundados em torno dos quais se agrupam interesses comuns de investigadores de diversos campos do conhecimento. [...] Ainda, organizam-se com a finalidade de sistematizar a experiência de pesquisa, desenvolver novos projetos, pautar a construção de novos planos de estudo, captar e organizar recursos humanos institucionais e técnicos ${ }^{12}$.

Com relação aos integrantes, a maioria dos grupos de pesquisa é composta por 1 a 17 membros, com diferentes níveis de qualificação e que desempenham papéis diferentes de trabalho, exibidos na tabela abaixo:

Tabela 3: Número de integrantes dos Grupos de Pesquisa em Enfermagem do Brasil no período de 2005 - 2007.

\begin{tabular}{|c|c|c|c|c|}
\hline $\begin{array}{c}\mathrm{N}^{\circ} \text { de } \\
\text { integrantes } \\
\text { dos GP }\end{array}$ & 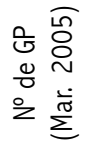 & 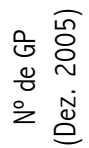 & 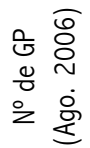 & 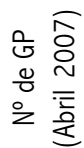 \\
\hline $1-17$ & 157 & 184 & 175 & 178 \\
\hline $18-34$ & 65 & 53 & 58 & 71 \\
\hline $35-51$ & 8 & 11 & 12 & 15 \\
\hline $52-68$ & 4 & 3 & 6 & 6 \\
\hline
\end{tabular}

Os perfis dos membros dos grupos de pesquisa são classificados pelo CNPq como pesquisadores, estudantes e técnicos, que estão organizados em torno da execução de linhas de pesquisa segundo uma regra hierárquica fundada na experiência e na competência técnico-científica.

Neste sentido, percebe-se aumento gradual da Iniciação Cientifica no país, que merece destaque. As bolsas de Iniciação Cientifica (IC) visam incentivar o ingresso e participação de jovens em grupos de pesquisa, preparando os jovens aptos a 
ingressar no mestrado e doutorado, e assim contribuindo para a diminuição do tempo de formação do pesquisador ${ }^{1}$.

A maioria dos grupos de pesquisa em Enfermagem no Brasil possui até 10 estudantes e 5 técnicos, sendo responsabilidade dos líderes de grupos designarem quais são os pesquisadores, estudantes e técnicos de seus grupos. Para ser classificado como estudante, é necessário que esteja matriculado em curso de graduação ou pós-graduação. Entretanto, compete aos dirigentes de pesquisa a identificação dos líderes e a certificação dos grupos de sua instituição'

A qualificação dos membros é visualizada na base de dados do CNPq como doutores, mestres, especialistas, graduandos e não-informados. A realidade nacional indica que os grupos de pesquisa possuem, em sua grande maioria, até 5 doutores, 3 mestres, 3 especialistas, 7 graduandos e 4 não-informados. 0s grupos de pesquisa constituídos de até 8 pesquisadores compõem $73 \%$ da realidade brasileira, conforme a tabela abaixo:

Tabela 4: Número de pesquisadores dos Grupos de Pesquisa em Enfermagem do Brasil no período de 2005 - 2007.

\begin{tabular}{|c|c|c|c|c|}
\hline $\begin{array}{c}\mathrm{N}^{0} \text { de } \\
\text { pesquisadores }\end{array}$ & 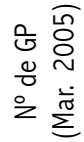 & 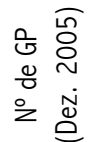 & 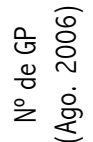 & 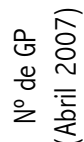 \\
\hline $1-8$ & 161 & 179 & 184 & 180 \\
\hline $9-16$ & 61 & 62 & 56 & 72 \\
\hline $17-24$ & 10 & 7 & 10 & 14 \\
\hline $25-32$ & 2 & 2 & 1 & 3 \\
\hline $33-40$ & - & - & 1 & 1 \\
\hline $41-47$ & - & 1 & 1 & - \\
\hline
\end{tabular}

Os pesquisadores desempenham papel fundamental no direcionamento do grupo de pesquisa, adequando os estudos à linha de pesquisa de forma estratégica e na orientação dos trabalhos junto aos membros. 0 papel desempenhado pelo pesquisador vai além da construção da informação, passando a ser construída, também, a formação, pois ele dá sustento à base educacional e compartilha habilidades adquirindo ao longo da carreira.

$\mathrm{Na}$ análise dos graduandos inseridos nos grupos de pesquisa, é percebido que a participação de acadêmicos ainda é baixa, demonstrando a necessidade de maiores estímulos desde o início da graduação, dada sua representatividade, conforme a tabela abaixo:

Tabela 5: Número de estudantes dos Grupos de Pesquisa em Enfermagem do Brasil no período de 2005 - 2007.

\begin{tabular}{|c|c|c|c|c|}
\hline $\begin{array}{c}\mathrm{N}^{0} \text { de } \\
\text { estudantes }\end{array}$ & 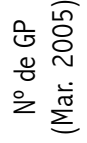 & 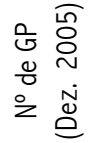 & 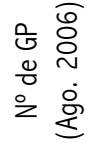 & 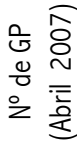 \\
\hline $0-10$ & 189 & 201 & 204 & 192 \\
\hline $11-21$ & 37 & 42 & 40 & 64 \\
\hline $22-32$ & 4 & 4 & 5 & 11 \\
\hline $33-43$ & 4 & 4 & 4 & 3 \\
\hline $44-54$ & - & - & - & 2 \\
\hline
\end{tabular}

Fonte: Base de Dados do Diretório dos Grupos de Pesquisa CNPq.
É importante salientar que 52 grupos de pesquisa de Enfermagem não contam com alunos de graduação em sua composição; este fato esclarece a presença do numeral zero no primeiro intervalo da primeira coluna da tabela apresentada. $A$ ausência de alunos de graduação nos grupos de pesquisa revela uma lacuna na formação destes indivíduos. É sabido o reflexo da experiência da pesquisa na prática profissional dos indivíduos, sendo que a não-exploração deste campo acarreta perdas significativas à qualidade do trabalho realizado pelo enfermeiro.

\section{CONSIDERAÇÕES FINAIS}

Os grupos de pesquisa constituem-se no lócus de produção de conhecimentos e formação de recursos humanos em pesquisa. No Brasil houve um crescimento significativo dos grupos de pesquisa da área da Enfermagem com o incremento da produção, qualificação dos integrantes, bem como 0 fortalecimento das bases de investigação e a maior visibilidade e reconhecimento da importância dos mesmos para o avanço da ciência, tecnologia e inovação da Enfermagem. Sem dúvida, o desenvolvimento da pesquisa é uma importante estratégia para o fortalecimento da Enfermagem como ciência e profissão.

Verifica-se que a base de dados do CNPq transforma-se em uma ferramenta captadora de pesquisadores. Uma vez que facilita contato com o líder do grupo pelo endereço eletrônico, sistematiza a busca e fornece informações aos interessados sobre o número e diversidade de temas trabalhados em cada grupo de pesquisa. Por esse motivo o registro dos grupos de pesquisa na base de dados do CNPq é fundamental para que a rede de conhecimento formada seja divulgada e visualizada.

$\mathrm{Na}$ evolução dos registros dos dados dos grupos de pesquisa na área da Enfermagem, nota-se uma tendência de melhor centralidade e clareza dos objetos de estudo, incluindo a redução do número de linhas de pesquisa e evitando a dispersão de temas, mostrando assim mais homogeneidade e delimitação dos campos de interesse.

Quanto à caracterização dos membros dos grupos de pesquisa, estes são constituídos por estudantes, pesquisadores e pessoal de apoio técnico. No entanto, a ausência de alunos de graduação em parte dos grupos de pesquisa pode revelar alguma dificuldade no registro dos mesmos, como não envolvimento dos pesquisadores em atividades de orientação em pesquisa, ou ainda, que pouco compreendem a importância do ensino do ponto de vista do acadêmico, de questionar as práticas conjuntamente com os estudantes, por fim, podem até estar desconsiderando a responsabilidade de formar com qualidade futuros pesquisadores. A outra grande perda é a do próprio acadêmico, futuro profissional.

Mesmo com o avanço na criação dos centros de excelência de produção de conhecimentos, nota-se que os grupos de pesquisas em Enfermagem ainda são uma forma organizativa incipiente, o que leva a demandar estratégias que priorizem e assegurem condições para o trabalho pesquisador nas instituições onde os mesmos se inserem. Também é importante avançar na visibilidade da produção de conhecimentos da área da Enfermagem. Isto desde o uso adequado de descritores específicos da área, como da abrangência dos conhecimentos 
produzidos por enfermeiros, de e para a Enfermagem, bem como da clareza dos objetos de conhecimento que são investigados e delimitados dentro do campo de conhecimento da Enfermagem. Contudo, é reconhecida a evolução dos programas de pós-graduação em Enfermagem no Brasil e das publicações pelos mesmos nos últimos trinta anos.

\section{Referências}

1. Conselho Nacional de Desenvolvimento Científico e TecnológicoCNPq. 0 CNPq. [citado 12 jun 2005] Disponível em: < http:// www.cnpq.br/cnpq/index.htm>

2 Coordenação de Aperfeiçoamento de Pessoal de Nível SuperiorCAPES. Avaliação. Programas da Área da Enfermagem. [citado set 2007]. Disponível em: <http://www.capes.gov.br/>

3. Erdmann AL, Mendes IAC, Leite JL. A enfermagem como área de conhecimento no CNPq: resgate histórico da representação de área. Esc Anna Nery Rev Enferm 2007 mar; 11 (1): 118-26.

4. Erdmann AL, Rodrigues RP, Silva IA, Fernandes JD, Santos RS, Araújo TL. A formação de doutores em enfermagem no Brasil. Texto \& Contexto Enferm 2002; 11(2): 66-76

5. Conselho Nacional de Desenvolvimento Científico e TecnológicoCNPq. Grupos de pesquisa: saiba mais. [citado 3 nov 2007]. Disponível em: http://www.cnpq.br/gpesq/saibamais.htm/>

6. Conselho Nacional de Desenvolvimento Científico e TecnológicoCNPq. Diretório dos Grupos de Pesquisa [base de dados on-line]. [citado 8 mar 2005]. Disponível em: <http://dgp.cnpq.br/ buscagrupo.htm>
Explorar as oportunidades de pesquisa, articulando políticas públicas e institucionais com fundos que mantenham e fortaleçam os estudos desenvolvidos pelos pesquisadores da Enfermagem, constitui-se uma importante estratégia para desenvolvimento social. E o CNPq vem incrementando a política de desenvolvimento científico, tecnológico e de inovação de nosso país com ganhos significativos para a área da Enfermagem.

7. Meirelles BHS, Erdmann AL. A interdisciplinaridade como construção do conhecimento em saúde e enfermagem. Texto \& Contexto Enferm [periódico on line] 2005; [citado 10 dez 2007]; 14(3): [aprox. 4 telas]. Disponível em: $<$ http://www.textoecontexto.ufsc.br/viewissue.php?id=12>

8. Castro MSL. A produção do conhecimento estimulada pela biblioteca universitária do ITPAC/FAHESA, no contexto do desenvolvimento regional do norte do Estado do Tocantins. Resumos apresentados no $14^{\circ}$ Seminário Nacional de Bibliotecas Universitárias; 2006 out 2227; Salvador (BA), Brasil. Brasília (DF): SNBU; 2000. p.7.

9. Costa RS, Carvalho DV. Análise da produção científica dos enfermeiros de Minas Gerais publicada em periódicos de enfermagem. Rev Latinoam Enfermagem 2001 set/out; 9 (5): 19-25.

10. Villar RMS. Produção do conhecimento em ergonomia na enfermagem [dissertação de mestrado]. Florianópolis (SC) Universidade Federal de Santa Catarina; 2002.

11. Oliveira A. Política científica no Brasil: análise das políticas de fomento à pesquisa do CNPq. [dissertação de mestrado] Florianópolis (SC): Universidade Federal de Santa Catarina; 2003.

12. Universidade Estadual de Campinas-UNICAMP. Faculdade de Educação. PAIDÉlA - Grupo de Estudos e Pesquisas em Filosofia da Educação. Os núcleos de pesquisa e as novas tendências da pós-graduação em educação. [on-line] [citado 9 dez 2007]. Disponível em: http://www.fae.unicamp.br/paideia. 